Supporting Information

\title{
Single-walled Carbon Nanotube Chiral Selectivity Exhibited by Commercially Available Hydrogels of Varying Composition
}

Sophia Zanoni, $\uparrow$ Brennan P. Watts $\uparrow$, and Kevin Tvrdy $\dagger^{*}$

tDepartment of Chemistry \& Biochemistry; University of Colorado, Colorado Springs; Colorado Springs, Colorado

*ktvrdy@uccs.edu 


\section{Table of contents}

$\underline{\text { Section }} \underline{\text { Title }}$ Page

S1. Detailed Materials and Methods..................................................S3

S2. Manufacturer specifications of gels investigated in this work.........S9

S3. Normalization of surface area presented by gels loaded into fritted columns .$S 10$

S4. Methods and quantitative results from per-SWNT-chirality absorbance spectra deconvolutions used in this work

S5. Intermediate experimental absorbance spectra used in calculation of differential absorbance spectra

S6. Results from 15-column iterative purification of SWNT using Sephacryl S200, Sephacryl S500, and Superose 6 .$S 18$

S7. Supporting Information References .$S 19$ 


\section{S1. Detailed Materials and Methods}

\section{S1.1 Materials and Equipment}

\section{S1.1.1 Hydrogels and hydrogel precursors}

- Sephacryl S-200 High Resolution (Sephacryl S200) - GE Healthcare Bio-Sciences AB; Lot \# 10240334

- Sephacryl S-400 High Resolution (Sephacryl S400) - Sigma Aldrich; Lot \# MKCG9384

- Sephacryl S-500 High Resolution (Sephacryl S500) - GE Healthcare Bio-Sciences AB

- Sephadex G-10 (Sephadex G10) - Aldrich Chemical; Lot \# 04901EY

- Sephadex G-100 (Sephadex G100) - GE Healthcare Bio-Sciences AB; Lot \# 10225511

- Sephadex G-200 (Sephadex G200) - Sigma Chemical; Lot \# 118B-0330

- CM Sephadex C-25 (CM Sephadex C25) - GE Healthcare Bio-Sciences AB; Lot \# 10057301

- SP Sephadex C-50 (SP Sephadex C50) - Pharmacia Fine Chemicals; Lot \# IF32029

- DEAE Sephadex A-25 (DEAE Sephadex A25) - Aldrich Chemical; Lot \# 07125JW

- QAE Sephadex A-25 (QAE Sephadex A25) - Sigma Chemical; Lot \# 118H0931

- Superdex 30 (Superdex 30) - GE Healthcare Bio-Sciences AB; Lot \# 10228261

- Superose 6 (Superose 6) - GE Healthcare Bio-Sciences AB; Lot \# 10241478

- Methylenebisacrylamide (MBA) - Sigma Aldrich; Lot \# MKBL2890V; 99\%

- Ammonium Persulfate (APS) - Fisher Scientific; Lot \# 197012

- Agarose (AGR) - TopVision Low Melting Point Agarose - Thermo Scientific; Lot 00269368

\section{S1.1.2 Other chemicals}

- Carbon Nanotubes - Sigma Aldrich; mfr. no. Signis ${ }^{\circledR}$ SG65i; single walled enriched in $(6,5)$ chirality ( $\geq 95 \%$ carbon basis [ $\geq 95 \%$ as carbon nanotubes]); Lot \# MKBZ1159V

- Sodium Dodecyl Sulfate (SDS) - Sigma Life Science; Lot\# SLBM8498V; $\geq 98.5 \%$

- Methanol - Fisher Chemical (Certified ACS); Lot \# 180816; $\geq 99.8 \%$

- Gas nitrogen - Airgas, Ultra High Purity

- Liquid nitrogen - Airgas, Nitrogen, Refrigerated Liquid, UN \# UN1977

- Deionized water - resistivity of $15 \mathrm{M} \Omega$

\section{S1.1.3 Equipment}

- Waring commercial blendor - Attach Series B Series \# 5012 G

- Mesh filters - SHANGHAI Yi Kai Electromechanical Technology

- Leica DM750 M Binocular Materials Microscope fitted with Leica ICC50 HD Microscope Camera

- Lyophilization System - SpeedVac SC110 vacuum concentrator connected to a Savant Instruments RVT4104- 120 cold trap at $-60^{\circ} \mathrm{C}$

- Carbon conductive tabs - PELCO Tabs

- SEM Specimen Mount - PELCO SEMClip ${ }^{\mathrm{TM}}, \varnothing 25 \mathrm{~mm}$ Pin Mount, 2 clips

- Gold sputterer - Denton Vacuum, Desk V

- Scanning electron microscope - TESCAN VEGA3 SBU 
- Thermo Scientific Disposable Plastic Columns (Number 29920); $7 \mathrm{~mm}$ inner diameter, $60 \mathrm{~mm}$ height; porous polyethylene disk ( $30 \mu \mathrm{m}$ pore size) used below the packed gel bed

- Tip horn Sonicator - Branson Digital Sonifier, Model 250, 1/2" Tip, coupled with $50 \mathrm{~mL}$ jacketed beaker for temperature stabilization during prolonged sonication

- Ultracentrifuge - Sorvall MX 120 Plus Micro-Ultracentrifuge with S50-ST Rotor

- 120v Digital Tube Revolver; Thermo Scientific

- HPAgilent 8453 G1103A Spectrometer; Agilent Technologies

\section{S1.2 Methods}

\section{S1.2.1 Generation of MBA and AGR-only hydrogel microparticles}

A hydrogel comprised of MBA radically polymerized by APS was generated by first dissolving $1.052 \mathrm{~g}$ of MBA within $18 \mathrm{~mL}$ of solvent comprised of a 3:1 mixture of DI water:methanol within a $50^{\circ} \mathrm{C}$ oil bath under vigorous stirring using a stir bar within a $20 \mathrm{~mL}$ vial capped with a septum. Upon full dissolution, the septum was pierced with two needles: 1 ) a nitrogen gas inlet needle was placed in the liquid; and 2) an outlet needle was placed in the headspace. The solution was degassed for 5 minutes while stirring to evacuate the system of oxygen both dissolved in the solution and in the headspace of the container. After degassing, the needles were removed and polymerization initiated by injection of $250 \mu \mathrm{L}$ of APS solution (also degassed) at concentration of $180 \mathrm{mg}$ APS per $\mathrm{mL}$ of 3:1 DI water:methanol through the septum. The solution was allowed to stir for 1 minute to fully incorporate the APS radical initiator after which the stirring was ceased and the vial stored in a $50^{\circ} \mathrm{C}$ oil bath during the gelation process, which began after approximately 5 minutes and was allowed to proceed overnight.

Additionally, efforts to synthesize a hydrogel comprised of aDEX radically polymerized by APS (in the absence of MBA) were made using a procedure similar to that used for the MBA/APS gel, with the exception that $1.036 \mathrm{~g}$ of aDEX (synthesized using previously reported methods ${ }^{1}$ )was added in place of the MBA. Following addition of APS, the gel was stored within a $50^{\circ} \mathrm{C}$ oil bath for 72 hours, after which the material became slightly more viscous but did not form a gel (remained in a liquid state), making this formulation unsuitable for processing and implementation within gel-based SWNT purification schemes.

A hydrogel comprised exclusively of AGR at concentration of $1.5 \%$ by weight in water was synthesized to afford analysis of its interaction with SWNT material in comparison with equal surface areas of other commercially available gels both with and without AGR. A volume of $15 \mathrm{~mL}$ of deionized water was loaded into a $20 \mathrm{~mL}$ vial and brought to a temperature of $95^{\circ} \mathrm{C}$ in an oil bath, to which $225 \mathrm{mg}$ of agarose powder was added and stirred for approximately one hour until fully dissolved. The vial was capped and stored at room temperature for 24 hours, after which the sample was further processed by blending and filtration to afford monodisperse microparticles suitable for use as SWNT purification material.

Hydrogels comprised exclusively of AGR at varying AGR concentration were generated by first heating $50 \mathrm{~mL}$ of DI water in a $100 \mathrm{~mL}$ round bottom flask connected to a water-cooled condenser along with a stir bar. Upon reflux, a total of $2.0 \mathrm{~g}$ of AGR powder was added and stirred vigorously for two hours. After fully dissolved, aliquots of the boiling AGR solution (at 4\% AGR concentration) were removed and mixed with neat boiling water by autopipette. A total of five gels were prepared, with approximate agarose concentrations of $0.5 \%, 1 \%, 2 \%, 3 \%$, and $4 \%$ weight by volume and total volumes of $15 \mathrm{~mL}$. For the $4 \%$ agarose gel, $15 \mathrm{~mL}$ of the mixture were drawn directly and placed in a $20 \mathrm{~mL}$ scintillation vial. For the $3 \%$, $2 \%, 1 \%$, and $0.5 \%$ gels $11.25,7.5,3.75$, and $1.88 \mathrm{~mL}$ respectively of $4 \%$ agarose solution were mixed with the corresponding volume of boiling water to afford a gel volume of $15 \mathrm{~mL}$. Vials containing liquified AGR solution were capped and stored at room temperature overnight to afford gelation and full thermal equilibration. 


\section{S1.2.2 Electron microscopy of hydrogel surfaces}

Hydrogels (as received from manufacturer) were loaded into fritted columns to afford approximately a $5 \mathrm{~mm}$ bed height and thrice rinsed with $1 \mathrm{~mL}$ aliquots of DI water to remove ethanol and generally cleanse the gels. Approximately $0.5 \mathrm{~g}$ of each gel was removed from the column and placed in a vial along with $0.5 \mathrm{~mL}$ of DI water. Vials were capped and contained suspensions homogenized by hand before flashfreezing within a liquid nitrogen bath. Vials were removed from the nitrogen bath, the lids removed and replaced with lids containing perforations, and placed within the chamber of a lyophilization system, where they were subject to vacuum for approximately 6 hours to completely remove water while persevering the micro- and nanoscale structure of the crosslinked gel matrix. Small amounts of powdered materials were transferred by spatula to carbon tape atop an SEM specimen mount with clips, which was sputtered with $\sim 10 \mathrm{~nm}$ gold and clips placed atop the areas of the carbon tape containing lyophilized gel microspheres. Electron microscopy images were collected near the crown of microspheres in close special proximity to clips to avoid charging effects, using electrons accelerated at $20 \mathrm{keV}$, minimal working distance, and detecting backscattered electrons.

\section{S1.2.3 Generation of SWNT suspension in aqueous SDS solution}

Suspensions of individualized SWNT were generated by tip horn sonication (14W, $1 / 2$ "tip) of a $30 \mathrm{~mL}$ solution of $1 \mathrm{mg}$ SWNT per $\mathrm{mL} 70 \mathrm{mM}$ SDS for 5.6 hours followed by ultracentrifugation $(252,721 \mathrm{k} \times \mathrm{g})$ for 2 hours and collection of the top $90 \%$ of each centrifuge tube. Enriched nanotubes were used in this study because of increased accuracy of spectral fitting/deconvolution while still demonstrating enhancement of chiral purity by hydrogel methods. For 'single gel-based SWNT purification' methods the SWNT suspension was pre-purified using Sephacryl S200 to remove amorphous carbon known to limit the adsorption and elution of SWNT materials in early columns of iterative purification ${ }^{1}$ and afford quantifiable purification of SWNT using a single interaction with gel. Additional information about the pre-purification process can be found in the supplementary materials of our previous work. ${ }^{1}$ For 'multi column gel-based SWNT purification' methods the post-ultracentrifugation SWNT suspension was used without dilution or pre-purification.

\section{S1.2.4 Single gel-based SWNT purification using column configuration}

A volume of aqueous suspension containing hydrogel microspheres (commercially sourced materials) or microparticles (lab synthesized materials) that collectively presented $125 \mathrm{~cm}^{2}$ of gel surface area was loaded into a fritted column, allowing excess water to pass under gravitational force. Thrice, approximately $1 \mathrm{~mL}$ of $70 \mathrm{mM}$ aqueous SDS solution was gently added to the top of each gel bed to afford its equilibration with equivalent surfactant concentration as that present in the SWNT suspension. Alongside the gel-containing columns, an additional control column containing a frit without gel was also prepared and 'equilibrated' with $70 \mathrm{mM}$ aqueous SDS to afford analysis of SWNT materials adsorbed to each gel by differential absorbance spectroscopy.

Pre-purified SWNT suspensions of peak optical density of approximately 2.5 in the nIR $E_{11}$ region of SWNT absorbance (1 cm path length) were used as pre-gel-interaction SWNT, which afforded spectroscopic characterization of post-gel-interaction SWNT suspensions without the need for additional dilution or spectroscopic cells of alternative path length. A total of $1.0 \mathrm{~mL}$ of SWNT suspension was gently added to the top of each gel bed (or frit, in the case of the gel-free control) and allowed to pass under gravitational force to a collection vessel beneath each column. Collected material was then passed a second time through each column to afford ample opportunity for SWNT to adsorb to each gel under overloading conditions. ${ }^{2}$ To remove SWNT materials from the interstitial space of both the gel bed and the frit, a total of $1.0 \mathrm{~mL}$ of $70 \mathrm{mM}$ aqueous SDS solution was added to the top of each column, allowed to flow through under gravitational force, and collected within each vial already containing SWNT 
suspensions that had twice passed through each column. The interstitial space of each column was then cleared and collected using slight overpressure applied by a syringe. Each aqueous suspension of postgel-interaction SWNT in 70mM SDS was capped and analyzed without dilution or other alteration using absorbance spectroscopy with a $1 \mathrm{~cm}$ path length cell.

To elute purified SWNT from each gel a total of $0.50 \mathrm{~mL}$ of $175 \mathrm{mM}$ aqueous SDS solution was gently added to the top of each gel bed and allowed to pass under gravitational force into a clean vial. The interstitial space of each column was then cleared and collected using slight overpressure applied by a syringe. Each aqueous suspension of post-gel-interaction SWNT in 175mM SDS was capped and analyzed without dilution or other alteration using absorbance spectroscopy with a $1 \mathrm{~cm}$ path length cell.

\section{S1.2.5 Single gel-based SWNT purification using centrifuge configuration}

In order to afford a quantitative comparison of SWNT adsorbed to and eluted from AGR-only gels of varying AGR content (ranging from 0.5-4.0 wt\%) it was necessary to utilize a centrifuge-based approach to afford phase separation between the gel and aqueous SWNT suspension instead of a frit-based column approach. This was due to the malleable nature of low-agarose-concentration gels and their tendency to clog porous frits, preventing SWNT flow through gel beds. Our group has previously demonstrated qualitative equivalencies between centrifuged-based and column-based gel-based SWNT purification methodologies, ${ }^{3}$ and we expect that the methods employed here afford elucidation of SWNT uptake and elution by gels of varying AGR concentration.

While the surface area of each of the five gels of varying AGR concentration $(0.5,1,2,3$, and 4 , wt\%) was not quantified in the same manner as the 14 gels constituting the primary portion of this study, the five gels were nonetheless processed identically and equivalent heights of gel were used, making the methods employed here suitable to elucidate trends in SWNT uptake and elution vs. the AGR content of gels. To afford similar particle size distribution among the gels, each AGR block $(0.5,1,2,3$, and 4 wt\%) totaling $\sim 15-18 \mathrm{~mL}$ within a $20 \mathrm{~mL}$ scintillation vial was roughly broken up with a spatula and transferred to the vessel of the blendor along with DI water for a total volume of $\sim 100 \mathrm{~mL}$. Each was blended for $30-$ 120 seconds on blendor setting between 3 and 6, noting that the higher-AGR-content gels were more rigid and required longer blending at higher speeds to achieve fractionation. Material from the blendor vessel was placed in small batches directly atop a wire mesh filter of $38 \mu \mathrm{m}$ aperture, with materials passing through directly to a wire mesh filter of $25 \mu \mathrm{m}$ aperture. Passage of materials was facilitated by injecting DI water and tapping/shaking the filter. Materials retained above the $25 \mu \mathrm{m}$ aperture filter were passed a second time through a system of (cleaned) $38 \mu \mathrm{m}$ aperture filter passing to $25 \mu \mathrm{m}$ aperture filter. Materials retained atop the $25 \mu \mathrm{m}$ aperture filter were retained, stored in DI water, and allowed to settle under gravitational force. DI water was added/removed to the supernatant to afford a suspension of approximately equal volumes of DI water and settled AGR particles.

Light microscope images of each microparticle suspension were collected and qualitatively compared to afford the assumption that the size distribution of particles within each suspension was equivalent, see Figure S3. Each suspension was homogenized and $1 \mathrm{~mL}$ added to the top of a fritted column of $7 \mathrm{~mm}$ inner diameter, allowing water to pass through under gravitational force. The height of each gel bed was measured with calipers, affording normalization of each material according to total volume of packed gel materials.

The equivalent amount of gel to afford a $6 \mathrm{~mm}$ packed gel height of each gel formulation $(0.5,1,2$, 3 , and $4 \mathrm{wt} \%$ ) was added to a $1.5 \mathrm{~mm}$ centrifuge tube, spun at $20 \mathrm{kxg}$, and decanted. Twice, $\sim 1 \mathrm{~mL}$ of aqueous $70 \mathrm{mM}$ SDS was added to each of the five vials (plus one control containing no gel), homogenized by hand, centrifuged at $20 \mathrm{k} \times \mathrm{g}$ for $3 \mathrm{~min}$, and decanted to waste to equilibrate the gel 
material with surfactant. A total of $1 \mathrm{~mL}$ of aqueous SWNT in $70 \mathrm{mM}$ SDS of peak optical density of $\sim 1.0$ was added to each vial, the contents of which were homogenized by either hand or touch mixer. Each was placed on an automated rotator for 10 minutes and subsequently centrifuged for 5 min at $20 \mathrm{kx} \mathrm{g}$. The supernatant of each tube was carefully decanted to a clean $7 \mathrm{~mL}$ vial. To each vial, a total of $1 \mathrm{~mL}$ neat $70 \mathrm{mM}$ SDS was added the contents of which were homogenized by either hand or touch mixer, centrifuged, and added to the SWNT suspension to double its volume to $2 \mathrm{~mL}$. These containers represented the post-gel-interaction SWNT suspension for each gel. A total of $0.5 \mathrm{~mL} 5 \mathrm{wt} \%$ was added to each vial, homogenized, and rotated for $10 \mathrm{~min}$. Each vial was centrifuged for $5 \mathrm{~min}$ at $20 \mathrm{kxg}$ and decanted into a clean vial for spectroscopic analysis. All solutions were analyzed without dilution or other alteration using absorbance spectroscopy with a $1 \mathrm{~cm}$ path length cell.

\section{S1.2.6 Multi column gel-based SWNT purification}

A multi column configuration was utilized to assess the chiral selectivity of select gels that exhibited significant elution efficiency (Sephacryl S200 and Superose 6) or reasonable elution efficiency and a fundamentally unique chiral-distribution of eluted SWNT materials (Sephacryl S500). For this work a stacked configuration akin to that reported by Kataura and coworkers ${ }^{2}$ was employed instead of an iterative configuration utilized in the past by us $^{1,3}$ and others ${ }^{4,5}$ because a stack sill affords identification of SWNT purification properties such as chiral selectivity and elution order while taking far less time to execute experimentally. For each of the three gels investigated (Sephacryl S200, Superose 6, and Sephacryl S500), equivalent amounts of hydrogel were loaded into each of 15 fritted columns of $7 \mathrm{~mm}$ inner diameter and equilibrated with surfactant by thrice adding $1 \mathrm{~mL}$ of $70 \mathrm{mM}$ aqueous SDS.

For a given gel formulation, the surface area presented within each column was constant for each of the 15 stacked columns, however, the amount of gel within each column was different for each gel formulation. Such differences were based on surface area calculations of each gel to obtain a total surface area of $125 \mathrm{~cm}^{2}$ across all 15 columns. Additionally, while the chiral distribution of the pre-gel-interaction SWNT suspension was consistent among the three gels investigated, the SWNT concentration of each of the three suspensions differed, with the absorbance spectrum of the pre-gel-interaction SWNT suspension shown in Figure S1 for each of the Sephacryl S200, Superose 6, and Sephacryl S500 gel stacks. Per-gelformulation adjustments of both gel surface area and SWNT concentration were necessary to ensure that overloading conditions were met over multiple columns of SWNT/gel interaction as well as to ensure that sufficient SWNT materials would be eluted from each gel bed to afford quantitative spectroscopic analysis. In other words, use of a consistent SWNT concentration across all formulations would result in depletion of SWNT after only a few columns by the Superose 6 stack because that material takes up much more SWNT than either Sephacryl S200 or Sephacryl S500, while Sephacryl S500 would not exhibit chiral selectivity because its gel would be so overloaded that each of the 15 columns would be exposed to a SWNT suspension of nearly identical chiral distribution. Additionally, use of equivalent presented surface area

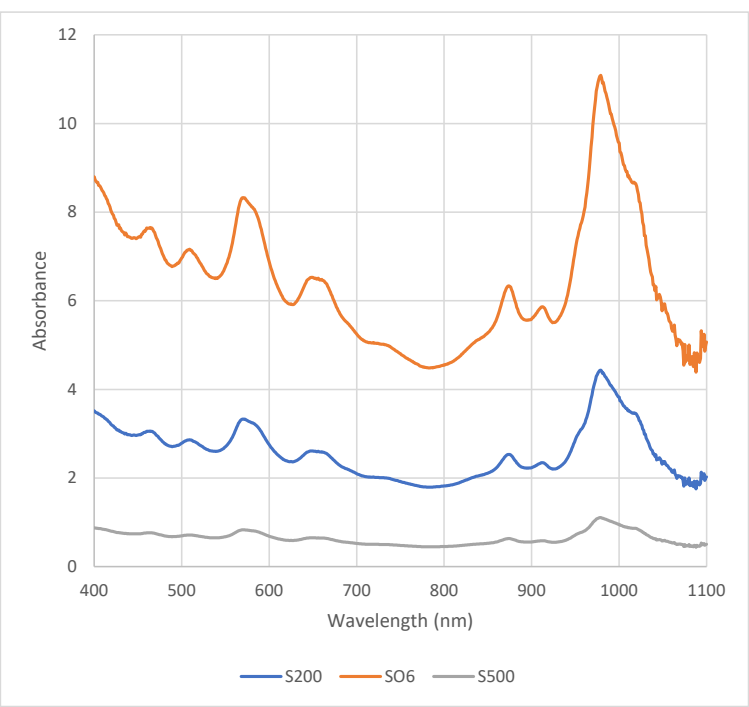

Figure S1. Absorbance spectra of SWNT solutions introduced to Sephacryl S200, Superose 6, and Sephacryl S500 within a multicolumn interaction scheme to generate data presented in Fig. 6 of the main body of this work. Absorbance reported at pathlength of $1 \mathrm{~cm}$ determined for Sephacryl S200 and S500 through dilution and mathematical correction. 
across all formulations may have provided sufficient elution of SWNT from Sephacryl S200 to afford quantitation of eluted materials by absorption spectroscopy, but an equivalent surface area of Sephacryl S500 both adsorbs and elutes far fewer SWNT, making quantitation of its elution potentially challenging. Adjustment of per-gel-formulation surface area (equivalent across all columns for a given formulation) and SWNT concentration of pre-gel-interaction suspension afford a qualitative comparison between three unique gel formations that exhibit vastly different chirality-dependent interactions with SWNT, the purpose of the experiment.

A total of 15 columns for each gel formulation were stacked such that material exiting the first SWNTgel interaction fed directly into the column containing the second gel bed, etc. A total of $3.6 \mathrm{~mL}$ of aqueous SWNT suspension in 70mM SDS was gently added to the top of the gel bed in the first column and allowed to flow through the stack of 15 columns under gravitational force. After SWNT suspension had completely passed through the uppermost column of a given stack (flow ceased), $0.5 \mathrm{~mL}$ of aqueous $70 \mathrm{mM}$ SDS was added to allow the SWNT present in the interstitial space of the gel and the frit (not adsorbed to the gel) to exit that column and pass through subsequent columns in the stack. Following passage of the $0.5 \mathrm{~mL}$ solution, the column was removed from the stack and an additional $1.0 \mathrm{~mL}$ of aqueous $70 \mathrm{mM}$ gently added to the top of the gel and collected as waste, ensuring the complete removal of any remaining SWNT and avoiding contamination of the elution suspension. After the SDS solution had completely passed through the column (flow ceased) a total of $0.5 \mathrm{~mL}$ of aqueous $175 \mathrm{mM} \mathrm{SDS}$ was gently added to the top of the gel bed, allowed to pass under gravitational force, and collected for spectroscopic analysis as SWNT eluted from each column of each gel formulation. All solutions were analyzed without dilution or other alteration using absorbance spectroscopy with a $1 \mathrm{~cm}$ path length cell. While elution suspensions were characterized for all 15 columns (for each of the three formulations), nearly all SWNT were removed from the system after 10 column for the Sephacryl S200 and Superose 6 materials and most of the SWNT removed from the system after 10 columns for the Sephacryl S500 material. Elution spectra and quantitative analysis thereof are presented in Fig. 6 of the main body (first 10 columns) and within Supporting Information Section S6 (all 15 columns). 


\section{S2. Manufacturer specifications of gels investigated in this work}

In Table S1, the reported manufacturer specifications on the original gel purpose, pore exclusion limit where applicable, and average particle size are listed. The exclusion limit is used here as it is reported for all of the gels, and represents the upper limit of the fractionation range of the gel. To estimate the pore size of each gel, reported in Fig. 1A, the exclusion limit, reported as a molecular weight, was converted to a protein hydrodynamic size using Erickson's equation to determine the smallest radius of a sphere that could contain the mass of a given protein, which is listed below as Eq. S1, ${ }^{6}$

$$
R_{\text {min }}=0.066 M^{1 / 3}
$$

where $\mathrm{M}$ is the protein mass in $\mathrm{Da}$. This estimation relies on the assumption that the pore diameter is equivalent to the diameter of the largest macromolecule that can enter it. There are a lot of different interpretations that can be made from the information here. It is all provided here in the interest of transparency, but has been condensed into the singular comparison of pore size, in the interest of concision.

\begin{tabular}{|l|l|l|l|}
\hline Gel & Original Purpose & Exclusion Limit (kDa) & $\begin{array}{l}\text { Average Particle Size } \\
(\mu \mathrm{m})\end{array}$ \\
\hline $\begin{array}{l}\text { Sephacryl S- } \\
200\end{array}$ & size exclusion chromatography & 400 & 50 \\
\hline $\begin{array}{l}\text { Sephacryl S- } \\
400\end{array}$ & size exclusion chromatography & 9000 & 50 \\
\hline $\begin{array}{l}\text { Sephacryl S- } \\
500\end{array}$ & size exclusion chromatography & 100000 & 35 \\
\hline $\begin{array}{l}\text { Sephadex G- } \\
10\end{array}$ & size exclusion chromatography & 0.7 & 110 \\
\hline $\begin{array}{l}\text { Sephadex G- } \\
100\end{array}$ & size exclusion chromatography & 150 & 205 \\
\hline $\begin{array}{l}\text { Sephadex G- } \\
200\end{array}$ & size exclusion chromatography & 200 & $\mathrm{n} / \mathrm{a}$ \\
\hline $\begin{array}{l}\text { CM } \\
\text { Sephadex C- } \\
25\end{array}$ & $\begin{array}{l}\text { ion exchange chromatography - } \\
\text { weak cation exchange gel }\end{array}$ & $\mathrm{n} / \mathrm{a}$ & $\mathrm{n} / \mathrm{a}$ \\
\hline $\begin{array}{l}\text { SP Sephadex } \\
\text { C-50 }\end{array}$ & $\begin{array}{l}\text { ion exchange chromatography - } \\
\text { strong cation exchange gel }\end{array}$ & $\mathrm{n} / \mathrm{a}$ & $\mathrm{n} / \mathrm{a}$ \\
\hline $\begin{array}{l}\text { DEAE } \\
\text { Sephadex A- } \\
25\end{array}$ & $\begin{array}{l}\text { ion exchange chromatography - } \\
\text { weak anion exchange gel }\end{array}$ & $\mathrm{n} / \mathrm{a}$ & $\mathrm{n} / \mathrm{a}$ \\
\hline $\begin{array}{l}\text { QAE } \\
\text { Sephadex A- } \\
25\end{array}$ & $\begin{array}{l}\text { ion exchange chromatography - } \\
\text { strong anion exchange gel }\end{array}$ & $\mathrm{n} / \mathrm{a}$ & $\mathrm{n} / \mathrm{a}$ \\
\hline Superdex 30 & size exclusion chromatography & 10 & 35 \\
\hline Superose 6 & size exclusion chromatography & 5000 & $\mathrm{n}$ \\
\hline
\end{tabular}

Table S1. Manufacturer specifications on gel properties, including the original purpose, pore exclusion limit where applicable, and the average particle size if reported. 


\section{S3. Normalization of surface area presented by gels loaded into fritted columns}

We have previously identified a direct correlation between hydrogel microsphere surface area and uptake of SWNT, ${ }^{3}$ information which has been used to afford quantitative comparison of hydrogels of differing formulation in terms of their uptake, retention, and elution of SWNT. ${ }^{1}$ Here, per-gel-formulation surface area was normalized through consideration of total hydrogel microsphere surface area per volume of homogenized solution, parameters utilized in our previous work to afford normalization of gel surface area $^{1}$ and visualized here within Figure S2, Where $V_{\text {tot. }}$ is the total volume of a homogenized or settled gel suspension, $V_{g}$ is the total volume of all packed gel particles within a settled gel suspension, and $V_{p . g}$ is the volume of the packed gel particles plus interstitial space around those packed particles within a settled

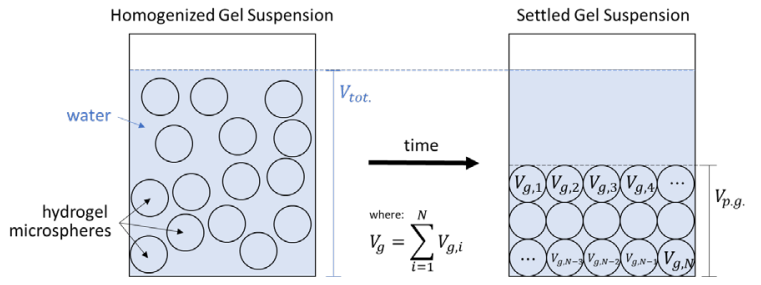

Figure S2. Depiction of the settling of hydrogel microspheres within a gel suspension over time, illustrating and interrelating total suspension volume to volume of packed gel and total hydrogel volume. gel suspension.

The relationship between the diameter of an individual particle within Figure $\mathbf{S 2}, d_{i}$, and individual particle volume depicted in Figure $\mathbf{S 2}, V_{g, i}$ is:

$$
V_{g, i}=\frac{4}{3} \pi\left(r_{i}\right)^{3}=\frac{4}{3} \pi\left(\frac{d_{i}}{2}\right)^{3}=\frac{\pi d_{i}^{3}}{6}
$$

while the surface area of an individual particle is:

$$
A_{i}=4 \pi\left(r_{i}\right)^{2}=4 \pi\left(\frac{d_{i}}{2}\right)^{2}=\pi d_{i}^{2} .
$$

The straightforward parameter to measure within the system is the total volume of a homogenized gel suspension, $V_{\text {tot. }}$, and the parameter of interest is the total hydrogel surface area among all the hydrogel microspheres containing within that hydrogel volume, $A_{t o t}$, such that $A_{t o t}=\sum_{i=1}^{N} A_{i}$. A logical ratio to express this parameter of interest is that of $A_{\text {tot. }} / V_{\text {tot. }}$, which can be expressed in terms of fraction of packed gel volume relative to homogenized gel suspension volume, $V_{p . g .} / V_{t o t}=f_{p . g .}$, along with the fraction of volume occupied by all hydrogel microsphere particles relative to that of the packed gel volume, $V_{g} / V_{p . g .}=f_{g e l}$ :

$$
\frac{A_{\text {tot. }}}{V_{\text {tot. }}}=\frac{V_{\text {p.g. }}}{V_{\text {tot. }}} \frac{V_{g}}{V_{p . g .}} \sum_{i=1}^{N} \frac{A_{i}}{V_{g, i}}=f_{\text {p.g. }} f_{\text {gel }} \sum_{i=1}^{N} \frac{6}{d_{i}}
$$

where the final equivalency was determined via substitution of Eqs. S2 and S3 within the term inside the sum. Because it is impractical to count each particle within an entire homogenized suspension, this analysis utilizes statistical methods based on microscope imaging and of a randomly selected group of hydrogel microspheres from a homogenized suspension of particles, affording the probability of observing a particle with diameter $d_{i}$ and diameter-space binning of $\Delta d_{i}, p\left(d_{i}\right) \Delta d_{i}$ such that the distribution is normalized: $\sum_{i=1}^{N} p\left(d_{i}\right) \Delta d_{i}=1$. This statistical analysis can be integrated within Eq. $S 4$ to yield:

$$
\frac{A_{\text {tot. }}}{V_{\text {tot. }}}=6 f_{p . g .} f_{\text {gel }} \sum_{i=1}^{N} \frac{p\left(d_{i}\right)}{d_{i}} \Delta d_{i}
$$

with the following dimensional analysis: 


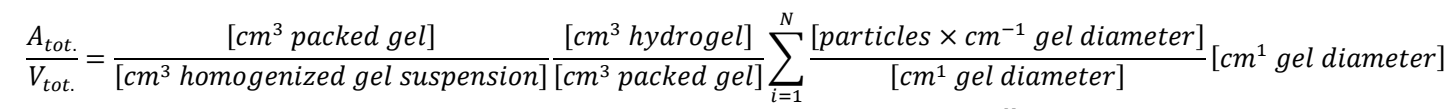

$$
\begin{aligned}
& =\frac{\left[\mathrm{cm}^{3} \text { hydrogel }\right]}{\left[\mathrm{cm}^{3} \text { homogenized gel suspension }\right]} \frac{1}{\left[\mathrm{~cm}^{1} \text { gel diameter }\right]} \sum_{i=1}^{N}[\text { particles }]= \\
& =\frac{\text { surface area of gel }\left[\mathrm{cm}^{2}\right]}{\text { volume of homogenized gel suspenions }\left[\mathrm{cm}^{3} \text { or } \mathrm{mL}\right]}
\end{aligned}
$$

where in the last equality $\left[\mathrm{cm}^{3}\right.$ hydrogel $]$ and $\left[\mathrm{cm}^{-1}\right.$ gel diameter $]$ combine to yield surface area of $\mathrm{gel}\left[\mathrm{cm}^{2}\right]$ because each are spatial dimensions of the physical gel particles.

Practical implementation of Eq. S5 for each homogenized gel suspension utilizes experimentally measured values of $f_{p . g}$. and a value of $f_{\text {gel }}=0.64$ assumed for irregular packing of spheres. ${ }^{7}$

To measure $f_{\text {p.g. }}$. of each gel it was first necessary to ensure that each gel was hydrated exclusively with water. Materials shipped wet in a mixture of ethanol and water (e.g. gels in the Sephacryl line) were washed by centrifugation at $5 \mathrm{kx}$ g such that, for a total of five iterations the gel was subjected to centrifugation with $\sim 10 \%$ of the centrifuge tube volume containing packed gel, decanted, and filled to maximum centrifuge tube volume with DI water. Materials shipped dry (e.g. DEAE A25) were hydrated with water according to the manufacturer's specifications. Each gel was allowed to settle under gravitational force and water removed/added such that the settled gel was approximately one third of the total volume. The $f_{p . g}$. of each gel suspension was measured by adding $1.0 \mathrm{~mL}$ of homogenized gel suspension to a fritted column of inner diameter $7 \mathrm{~mm}$ with column tip removed to expose the bottom portion of the frit. Excess water was allowed to flow through the frit and exit the column under gravitational force and the bottom of the frit patted dry, leaving water in three locations: within each gel microsphere, within the interstitial space above the frit and between microspheres (capillary force assumed to retain this water), and within the porous space of the frit (capillary force assumed to retain this water). Mass measurement of the column before (dry) and after (with gel, accounting for $52.8 \mu \mathrm{L}$ of water contained within each frit), along with the assumption that the density of each hydrated gel bead is equivalent to that of water $(1 \mathrm{mg} / \mathrm{mL}$ ), allowed for the determination (by mass) of the ratio of volume of settled gel per volume of homogenized gel, or $f_{p . g}$. As an alternative to the "by mass" method, the height of each gel bed (after water removed through fit under gravitational force) was measured by calipers, combined with the known dimensions of the column, and used to estimate (by volume) a value of $f_{p . g .}$. for each gel. Values determined by mass and by volume were compared to ensure accuracy and the average of the two used in Eqn. S5.

To determine the actual volume needed for a SWNT interaction, one needs to take into account the concentration and volume of SWNT within the suspension. Typically, one may use approximately $100 \mu \mathrm{L}$ of packed Sephacryl S200 gel per mL of SWNT and then make adjustments from that starting point. Here, it is sensible to calculate the volume of homogenized gel suspension needed to pack a column with 100 $\mu \mathrm{L}$ of Sephacryl S200 and then determine the surface area of that gel, then normalize all other gels to that surface area. Specifically, $100 \mu \mathrm{L}(0.100 \mathrm{~mL})$ of packed Sephacryl $\mathrm{S} 200$ contains $0.1 \mathrm{~mL}\left(f_{\text {gel }}\right)=$ $0.1 \mathrm{~cm}^{3}(0.64)=0.064 \mathrm{~mL}$ total volume of hydrogel microspheres, which exhibit a total surface area per gel volume given by considering application of statistical methods to Eq. S5:

$$
\frac{A_{\text {tot. }}}{V_{g}}=6 \sum_{i=1}^{N} \frac{p\left(d_{i}\right)}{d_{i}} \Delta d_{i}
$$


which, when applied to the probability distribution measured for Sephacryl S200, yields $1950 \mathrm{~cm}^{2}$ gel surface area per $\mathrm{mL}$ total volume of hydrogel microspheres. Combining this with the above calculation determines that a gel bed containing $100 \mu \mathrm{L}(0.100 \mathrm{~mL})$ of packed Sephacryl S200 exhibits $124.8 \mathrm{~cm}^{2}$ of hydrogel microsphere surface area, which was used as the per-gel-material surface area for all single SWNT/gel interactions employed here. Calculated parameters used in this study to ensure consistent surface area across the 14 gels of different formulation are listed in Table S2, while representative light microscope images and histograms showing particle size distribution for each of the gels are shown in

Figure S3.

\begin{tabular}{|l|l|l|l|l|}
\hline \multicolumn{1}{|c|}{ Gel } & \multicolumn{1}{|c|}{$\begin{array}{c}\text { Average } \\
\text { microsphere } \\
\text { diameter }(\boldsymbol{\mu m}) \pm \mathbf{1} \\
\text { standard deviation }\end{array}$} & $\begin{array}{c}\text { Surface area }\left(\mathbf{c m}^{2}\right) \text { per } \\
\text { volume homogenized } \\
\text { gel suspension }(\mathbf{m L})\end{array}$ & $\begin{array}{c}\text { Volume homogenized } \\
\text { gel suspension to } \\
\text { achieve 124.8 } \mathbf{c m}^{2} \text { gel } \\
\text { surface area }\end{array}$ & $\begin{array}{c}\text { Estimated gel bed } \\
\text { height in column of } \\
\text { 0.7 cm inner } \\
\text { diameter }\end{array}$ \\
\hline Sephacryl S-200 & $34.0 \pm 10.0$ & $432 \mathrm{~cm}^{2} / \mathrm{mL}$ & $289 \mu \mathrm{L}$ & $2.6 \mathrm{~mm}$ \\
\hline Sephacryl S-400 & $39.5 \pm 11.9$ & $356 \mathrm{~cm}^{2} / \mathrm{mL}$ & $350 \mu \mathrm{L}$ & $3.0 \mathrm{~mm}$ \\
\hline Sephacryl S-500 & $40.1 \pm 10.4$ & $367 \mathrm{~cm}^{2} / \mathrm{mL}$ & $340 \mu \mathrm{L}$ & $3.1 \mathrm{~mm}$ \\
\hline DEAE Sephadex A-25 & $71.6 \pm 21.4$ & $230 \mathrm{~cm}^{2} / \mathrm{mL}$ & $542 \mu \mathrm{L}$ & $4.9 \mathrm{~mm}$ \\
\hline QAE Sephadex A-25 & $89.9 \pm 23.2$ & $259 \mathrm{~cm}^{2} / \mathrm{mL}$ & $482 \mu \mathrm{L}$ & $6.9 \mathrm{~mm}$ \\
\hline CM Sephadex C-25 & $104.0 \pm 22.9$ & $145 \mathrm{~cm}^{2} / \mathrm{mL}$ & $859 \mu \mathrm{L}$ & $8.4 \mathrm{~mm}$ \\
\hline SP Sephadex C-50 & $191.6 \pm 43.3$ & $66 \mathrm{~cm}^{2} / \mathrm{mL}$ & $1.891 \mathrm{~mL}$ & $15.2 \mathrm{~mm}$ \\
\hline Sephadex G-10 & $52.1 \pm 14.4$ & $268 \mathrm{~cm}^{2} / \mathrm{mL}$ & $465 \mu \mathrm{L}$ & $3.9 \mathrm{~mm}$ \\
\hline Sephadex G-100 & $166.3 \pm 64.2$ & $116 \mathrm{~cm}^{2} / \mathrm{mL}$ & $1.071 \mathrm{~mL}$ & $12.1 \mathrm{~mm}$ \\
\hline Sephadex G-200 & $46.7 \pm 26.0$ & $478 \mathrm{~cm}^{2} / \mathrm{mL}$ & $261 \mu \mathrm{L}$ & $2.8 \mathrm{~mm}$ \\
\hline Superose 6 & $26.0 \pm 4.6$ & $539 \mathrm{~cm}^{2} / \mathrm{mL}$ & $232 \mu \mathrm{L}$ & $2.1 \mathrm{~mm}$ \\
\hline Superdex 30 & $26.0 \pm 5.7$ & $612 \mathrm{~cm}^{2} / \mathrm{mL}$ & $204 \mu \mathrm{L}$ & $2.1 \mathrm{~mm}$ \\
\hline AGR & $54.6 \pm 14.1$ & $331 \mathrm{~cm}^{2} / \mathrm{mL}$ & $377 \mu \mathrm{L}$ & $4.3 \mathrm{~mm}$ \\
\hline MBA-APS & $35.1 \pm 9.4$ & $129 \mathrm{~cm}^{2} / \mathrm{mL}$ & $970 \mu \mathrm{L}$ & $2.7 \mathrm{~mm}$ \\
\hline
\end{tabular}

Table S2. Measured and calculated parameters used to ensure that each gel bed (of differ gel formulation and gel particle size/distribution) exhibited normalized surface area of $125 \mathrm{~cm}^{2}$. 

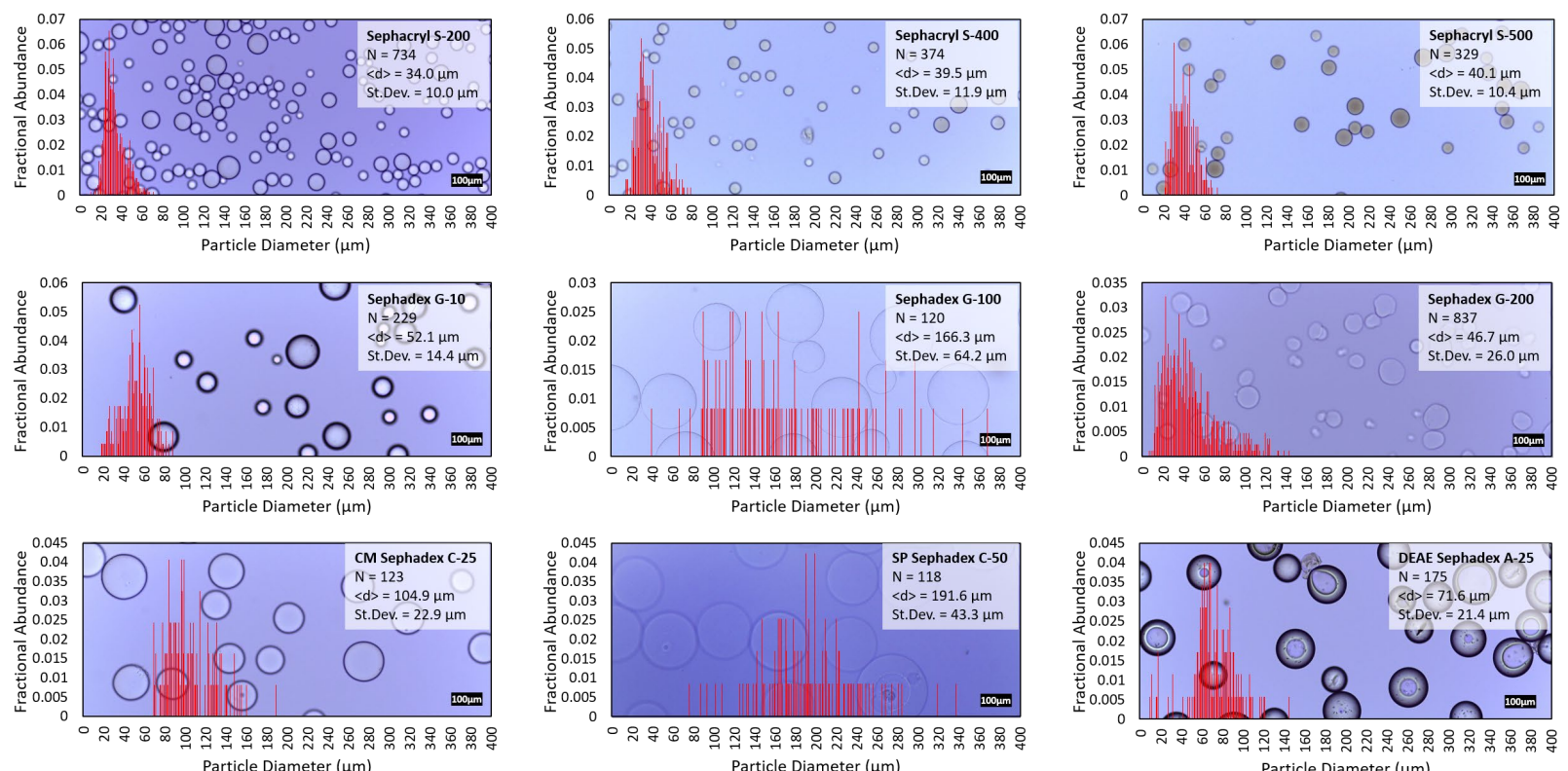

Particle Diameter $(\mu \mathrm{m})$

Particle Diameter $(\mu \mathrm{m})$
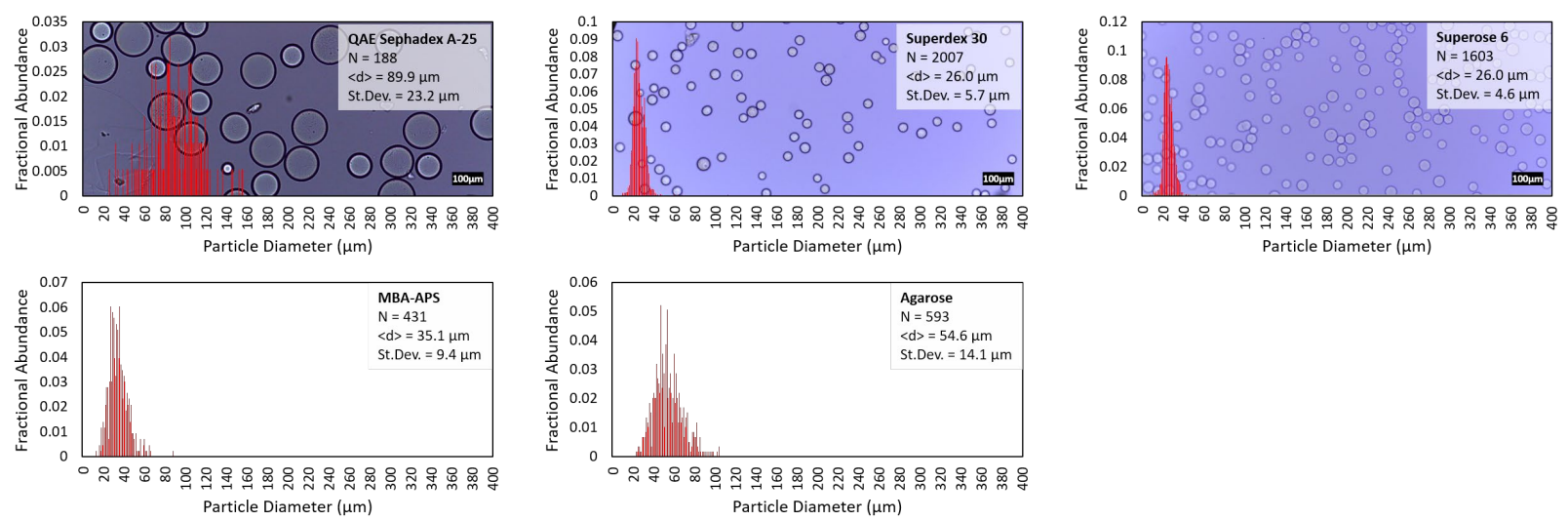

Figure S3. Histograms showing particle size distribution for each of the gels investigated in this work along with representative light microscope image used to generate particle size distributions. 


\section{S4. Methods and quantitative results from per-SWNT-chirality absorbance spectra deconvolutions used in this work}

For this work we employed a previously described method for the deconvolution of SWNT absorbance spectra and differential absorbance spectroscopy to afford per-SWNT-chirality quantitation. ${ }^{1,3}$ In brief, in house developed software (written in Mathematica) was used to identify the least squares best-fit in energy-space between a given experimental absorbance spectrum and a sum of simulated absorbance profiles of SWNT and an amorphous carbon background. In the case of best-fitting differential absorbance spectra, experimental spectra in the form of differential absorbance spectra (obtained via subtraction of experimental spectra) were used. All fitting is performed exclusively within the lowest energy $\left(E_{11}\right)$ region of the SWNT absorbance spectrum, which for the chiralities present within the SWNT suspensions employed in this work, is entirely captured within the region of $800-1100 \mathrm{~nm}(1.13-1.55 \mathrm{eV}) .{ }^{1}$

The employed software uses a linear background to account for the absorbance of amorphous carbon within this spectral region. ${ }^{8}$ Chirality specific SWNT absorbance is modeled in the $E_{11}$ region by a sum of Lorentzian line shapes that account for both the primary $\left(E_{11}\right)$ and phonon sideband (PSB) of the lowest energy SWNT electronic transition:

$$
\operatorname{Abs}_{(n, m)}(E)=\left(I_{E_{11}}\right)_{(n, m)} \frac{\gamma_{E_{11}}^{2}}{\left(E-E_{0(n, m)}\right)^{2}+\gamma_{E_{11}}^{2}}+R_{P S B / E_{11}}\left(I_{E_{11}}\right)_{(n, m)} \frac{\gamma_{P S B}^{2}}{\left(E-E_{0(n, m)}-E_{P S B}\right)^{2}+\gamma_{P S B}^{2}}
$$

where $\left(I_{E_{11}}\right)_{(n, m)}$ is the chirality $(n, m)$ depdendent intensity of the $E_{11}$ transition at its spectral center, $E_{0}(n, m)$, and $\gamma_{E_{11}}$ is a parameter related to the width of the simulated line shape of the $E_{11}$ transition. Further, each $\mathrm{E}_{11}$ transition has an accompanying PSB, which is red shifted by $E_{P S B}$ relative to the $\mathrm{E}_{11}$ peak, has width $\gamma_{P S B}$, and height relative to that of the $\mathrm{E}_{11}$ transition of $R_{P S B / E_{11}}$.

For a given fit employed in this work, seven functions of form Eq. S7 were summed to represent the seven chiralities $(10,2),(7,5),(6,5),(7,3),(8,3),(9,1)$, and $(6,4)$ present within the SWNT suspensions utilized. Parameters independent of SWNT chirality, $\gamma_{E_{11}}^{2}, \gamma_{P S B}^{2}, R_{P S B / E_{11}}$, and $E_{P S B}$ were fit globally across chiralities, while the chirality dependent center of each $E_{11}$ transition, $E_{0}(n, m)$, was held constant according to established chirality dependent optical properties of SWNT. ${ }^{9}$ The best-fit then yields identification of $\left(I_{E_{11}}\right)_{(n, m)}$ for each chirality, information which, when combined with chirality specific molar absorptivities, ${ }^{10}$ the assumption that each SWNT is approximately $300 \mathrm{~nm}$ in length following prolonged sonication, ${ }^{11}$ and knowledge of the SWNT suspension volume, affords specification of absolute number of each SWNT chirality present within a given suspension.

The total number of each SWNT chirality and per chirality fractional abundance for each SWNT suspension analyzed in this work are listed in Tables S3, S4, and S5. Persons interested in using the Mathematica script described here to fit absorbance spectra of SWNT suspension are encouraged to contact the corresponding author Kevin Tvrdy at ktvrdy@uccs.edu, who will freely provide the most updated version of this software.

\begin{tabular}{|l|c|c|}
\hline$(\boldsymbol{n}, \boldsymbol{m})$ & \# SWNT $(\boldsymbol{n}, \boldsymbol{m})$ & $\begin{array}{l}\text { Fractional } \\
\text { abundance }\end{array}$ \\
\hline$(10,2)$ & $1.53 \times 10^{15}$ & 0.0392 \\
\hline$(7,5)$ & $1.39 \times 10^{16}$ & 0.356 \\
\hline$(6,5)$ & $6.59 \times 10^{15}$ & 0.169 \\
\hline$(7,3)$ & $1.13 \times 10^{16}$ & 0.290 \\
\hline$(8,3)$ & $3.00 \times 10^{15}$ & 0.0767 \\
\hline$(9,1)$ & $2.72 \times 10^{15}$ & 0.0697 \\
\hline$(6,4)$ & $9.31 \times 10^{11}$ & $2.38 \times 10^{-5}$ \\
\hline Total & $3.91 \times 10^{16}$ & 1.00 \\
\hline
\end{tabular}

Table S3. Results of spectral deconvolution of SWNT suspension not subjected to gel interaction. 


\begin{tabular}{|l|c|c|l|l|l|c|}
\hline & \multicolumn{2}{|l|}{ Sephacryl S-200 } & \multicolumn{2}{l|}{ Sephacryl S-400 } & \multicolumn{2}{l|}{ Sephacryl S-500 } \\
\hline$(\boldsymbol{n}, \boldsymbol{m})$ & \# SWNT $(\boldsymbol{n}, \boldsymbol{m})$ & $\begin{array}{l}\text { Fractional } \\
\text { abundance }\end{array}$ & $\begin{array}{l}\text { \# SWNT } \\
(\boldsymbol{n}, \boldsymbol{m})\end{array}$ & $\begin{array}{l}\text { Fractional } \\
\text { abundance }\end{array}$ & $\begin{array}{l}\text { \# SWNT } \\
(\boldsymbol{n}, \boldsymbol{m})\end{array}$ & $\begin{array}{l}\text { Fractional } \\
\text { abundance }\end{array}$ \\
\hline$(10,2)$ & $3.11 \times 10^{12}$ & 0.000466 & $1.71 \times 10^{14}$ & 0.0555 & $2.48 \times 10^{11}$ & 0.000154 \\
\hline$(7,5)$ & $1.36 \times 10^{15}$ & 0.204 & $5.55 \times 10^{14}$ & 0.180 & $3.30 \times 10^{14}$ & 0.205 \\
\hline$(6,5)$ & $3.76 \times 10^{15}$ & 0.563 & $1.43 \times 10^{15}$ & 0.463 & $7.62 \times 10^{14}$ & 0.473 \\
\hline$(7,3)$ & $1.10 \times 10^{15}$ & 0.165 & $7.11 \times 10^{14}$ & 0.230 & $6.94 \times 10^{11}$ & 0.000430 \\
\hline$(8,3)$ & $1.54 \times 10^{13}$ & 0.00230 & $3.09 \times 10^{12}$ & 0.00100 & $2.92 \times 10^{14}$ & 0.181 \\
\hline$(9,1)$ & $1.47 \times 10^{14}$ & 0.0221 & $3.89 \times 10^{13}$ & 0.0126 & $1.27 \times 10^{14}$ & 0.0786 \\
\hline$(6,4)$ & $2.87 \times 10^{14}$ & 0.0430 & $1.78 \times 10^{14}$ & 0.0576 & $1.00 \times 10^{14}$ & 0.0621 \\
\hline Total & $6.67 \times 10^{15}$ & 1.00 & $3.09 \times 10^{15}$ & 1.00 & $1.61 \times 10^{15}$ & 1.00 \\
\hline
\end{tabular}

\begin{tabular}{|l|c|c|l|l|l|c|}
\hline & \multicolumn{3}{|l|}{ MBA Only (synthesized) } & \multicolumn{2}{l|}{ Sephadex G-10 } & \multicolumn{2}{l|}{ Sephadex G-100 } \\
\hline$(\boldsymbol{n}, \boldsymbol{m})$ & \# SWNT $(\boldsymbol{n}, \boldsymbol{m})$ & $\begin{array}{l}\text { Fractional } \\
\text { abundance }\end{array}$ & $\begin{array}{l}\text { \# SWNT } \\
(\boldsymbol{n}, \boldsymbol{m})\end{array}$ & $\begin{array}{l}\text { Fractional } \\
\text { abundance }\end{array}$ & $\begin{array}{l}\text { \# SWNT } \\
(\boldsymbol{n}, \boldsymbol{m})\end{array}$ & $\begin{array}{l}\text { Fractional } \\
\text { abundance }\end{array}$ \\
\hline$(10,2)$ & $2.63 \times 10^{14}$ & 0.0978 & $4.70 \times 10^{14}$ & 0.0670 & $2.08 \times 10^{14}$ & 0.0510 \\
\hline$(7,5)$ & $6.64 \times 10^{14}$ & 0.240 & $2.19 \times 10^{15}$ & 0.312 & $1.22 \times 10^{15}$ & 0.298 \\
\hline$(6,5)$ & $9.85 \times 10^{14}$ & 0.366 & $1.30 \times 10^{15}$ & 0.185 & $1.10 \times 10^{15}$ & 0.269 \\
\hline$(7,3)$ & $5.95 \times 10^{14}$ & 0.221 & $1.86 \times 10^{14}$ & 0.265 & $8.09 \times 10^{14}$ & 0.198 \\
\hline$(8,3)$ & $1.06 \times 10^{14}$ & 0.0394 & $7.88 \times 10^{14}$ & 0.112 & $2.81 \times 10^{14}$ & 0.0687 \\
\hline$(9,1)$ & $8.43 \times 10^{13}$ & 0.0313 & $4.08 \times 10^{14}$ & 0.0581 & $2.87 \times 10^{14}$ & 0.0702 \\
\hline$(6,4)$ & $1.44 \times 10^{13}$ & 0.00535 & $5.03 \times 10^{14}$ & $7.16 \times 10^{-5}$ & $1.81 \times 10^{14}$ & 0.0443 \\
\hline Total & $2.69 \times 10^{15}$ & 1.00 & $7.03 \times 10^{15}$ & 1.00 & $4.09 \times 10^{15}$ & 1.00 \\
\hline
\end{tabular}

\begin{tabular}{|l|c|c|l|c|l|c|}
\hline & \multicolumn{2}{|l|}{ Sephadex G-200 } & \multicolumn{2}{l|}{ CM Sephadex C-25 } & \multicolumn{2}{l|}{ SP Sephadex C-50 } \\
\hline$(\boldsymbol{n}, \boldsymbol{m})$ & \# SWNT $(\boldsymbol{n}, \boldsymbol{m})$ & $\begin{array}{l}\text { Fractional } \\
\text { abundance }\end{array}$ & $\begin{array}{l}\text { \# SWNT } \\
(\boldsymbol{n}, \boldsymbol{m})\end{array}$ & $\begin{array}{l}\text { Fractional } \\
\text { abundance }\end{array}$ & $\begin{array}{l}\text { \# SWNT } \\
(\boldsymbol{n}, \boldsymbol{m})\end{array}$ & $\begin{array}{l}\text { Fractional } \\
\text { abundance }\end{array}$ \\
\hline$(10,2)$ & $2.76 \times 10^{14}$ & 0.222 & $3.20 \times 10^{12}$ & 0.00624 & $3.58 \times 10^{14}$ & 0.443 \\
\hline$(7,5)$ & $1.74 \times 10^{14}$ & 0.140 & $9.71 \times 10^{13}$ & 0.189 & $1.55 \times 10^{11}$ & 0.000192 \\
\hline$(6,5)$ & $3.48 \times 10^{14}$ & 0.280 & $2.73 \times 10^{14}$ & 0.531 & $1.30 \times 10^{12}$ & 0.00160 \\
\hline$(7,3)$ & $3.77 \times 10^{14}$ & 0.304 & $1.08 \times 10^{14}$ & 0.210 & $4.48 \times 10^{14}$ & 0.554 \\
\hline$(8,3)$ & $5.13 \times 10^{13}$ & 0.0413 & $1.94 \times 10^{12}$ & 0.00378 & $5.54 \times 10^{11}$ & 0.000685 \\
\hline$(9,1)$ & $7.80 \times 10^{12}$ & 0.00628 & $9.92 \times 10^{12}$ & 0.0193 & $5.61 \times 10^{10}$ & $6.94 \times 10^{-5}$ \\
\hline$(6,4)$ & $7.40 \times 10^{12}$ & 0.005961 & $2.07 \times 10^{13}$ & 0.0403 & $3.96 \times 10^{11}$ & 0.000491 \\
\hline Total & $1.24 \times 10^{15}$ & 1.00 & $5.13 \times 10^{14}$ & 1.00 & $8.08 \times 10^{14}$ & 1.00 \\
\hline
\end{tabular}

Table S4. Results of spectral deconvolution of SWNT suspensions following gel interaction (passed through gel, not eluted from gel), for Sephacryl S-200, Sephacryl S-400, Sephacryl S-500, MBA Only (synthesized), Sephadex G-10, Sephadex G-100, Sephadex G-200, CM Sephadex C-25, and SP Sephadex C-50. 


\begin{tabular}{|l|c|c|l|l|l|c|}
\hline & \multicolumn{3}{|l|}{ DEAE Sephadex A-25 } & \multicolumn{2}{l|}{ QAE Sephadex A-25 } & \multicolumn{2}{l|}{ Superdex 30 } \\
\hline $\mathbf{( n , \boldsymbol { m } )}$ & \# SWNT $(\boldsymbol{n}, \boldsymbol{m})$ & $\begin{array}{l}\text { Fractional } \\
\text { abundance }\end{array}$ & $\begin{array}{l}\text { \# SWNT } \\
(\boldsymbol{n}, \boldsymbol{m})\end{array}$ & $\begin{array}{l}\text { Fractional } \\
\text { abundance }\end{array}$ & $\begin{array}{l}\text { \# SWNT } \\
(\boldsymbol{n}, \boldsymbol{m})\end{array}$ & $\begin{array}{l}\text { Fractional } \\
\text { abundance }\end{array}$ \\
\hline$(10,2)$ & $8.50 \times 10^{10}$ & $7.36 \times 10^{-5}$ & $9.52 \times 10^{10}$ & $2.03 \times 10^{-5}$ & $2.34 \times 10^{14}$ & 0.201 \\
\hline$(7,5)$ & $3.57 \times 10^{13}$ & 0.0309 & $1.24 \times 10^{15}$ & 0.265 & $1.54 \times 10^{14}$ & 0.132 \\
\hline$(6,5)$ & $4.47 \times 10^{14}$ & 0.387 & $1.22 \times 10^{15}$ & 0.261 & $2.42 \times 10^{14}$ & 0.208 \\
\hline$(7,3)$ & $4.46 \times 10^{11}$ & 0.000386 & $1.15 \times 10^{15}$ & 0.246 & $4.97 \times 10^{14}$ & 0.427 \\
\hline$(8,3)$ & $3.27 \times 10^{14}$ & 0.283 & $5.51 \times 10^{14}$ & 0.118 & $1.02 \times 10^{13}$ & 0.00874 \\
\hline$(9,1)$ & $2.04 \times 10^{14}$ & 0.177 & $3.96 \times 10^{14}$ & 0.0843 & $4.92 \times 10^{12}$ & 0.00423 \\
\hline$(6,4)$ & $1.41 \times 10^{14}$ & 0.122 & $1.19 \times 10^{14}$ & 0.0254 & $2.13 \times 10^{13}$ & 0.0183 \\
\hline Total & $1.16 \times 10^{15}$ & 1.00 & $4.68 \times 10^{15}$ & 1.00 & $1.16 \times 10^{15}$ & 1.00 \\
\hline
\end{tabular}

\begin{tabular}{|l|c|c|l|c|}
\hline & \multicolumn{2}{|l|}{ Superose $\mathbf{6}$} & \multicolumn{2}{l|}{ Agarose Only (synthesized) } \\
\hline$(\boldsymbol{n}, \boldsymbol{m})$ & \# SWNT $(\boldsymbol{n}, \boldsymbol{m})$ & $\begin{array}{l}\text { Fractional } \\
\text { abundance }\end{array}$ & $\begin{array}{l}\text { \# SWNT } \\
(\boldsymbol{n}, \boldsymbol{m})\end{array}$ & $\begin{array}{l}\text { Fractional } \\
\text { abundance }\end{array}$ \\
\hline$(10,2)$ & $5.51 \times 10^{14}$ & 0.0332 & $9.63 \times 10^{13}$ & 0.0488 \\
\hline$(7,5)$ & $5.26 \times 10^{15}$ & 0.317 & $4.85 \times 10^{14}$ & 0.245 \\
\hline$(6,5)$ & $3.81 \times 10^{15}$ & 0.230 & $5.69 \times 10^{14}$ & 0.288 \\
\hline$(7,3)$ & $3.83 \times 10^{15}$ & 0.231 & $5.62 \times 10^{13}$ & 0.0285 \\
\hline$(8,3)$ & $2.12 \times 10^{15}$ & 0.128 & $5.29 \times 10^{14}$ & 0.268 \\
\hline$(9,1)$ & $1.03 \times 10^{15}$ & 0.0617 & $1.63 \times 10^{14}$ & 0.0826 \\
\hline$(6,4)$ & $4.90 \times 10^{11}$ & $2.95 \times 10^{-5}$ & $7.64 \times 10^{13}$ & 0.0387 \\
\hline Total & $1.66 \times 10^{16}$ & 1.00 & $1.97 \times 10^{15}$ & 1.00 \\
\hline
\end{tabular}

Table S5. Results of spectral deconvolution of SWNT suspensions following gel interaction (passed through gel, not eluted from gel), for DEAE Sephadex A-25, QAE Sephadex A-25, Superdex 30, Superose 6, and Agarose Only (synthesized). 


\section{S5. Intermediate experimental absorbance spectra used in calculation of differential absorbance spectra}

All the spectra used in the calculation of the differential absorbance spectra are shown in Figure S4. These include the control sample and the spectra of the SWNT after passing through and interacting with a gel bed, for each of the 14 gels studied.

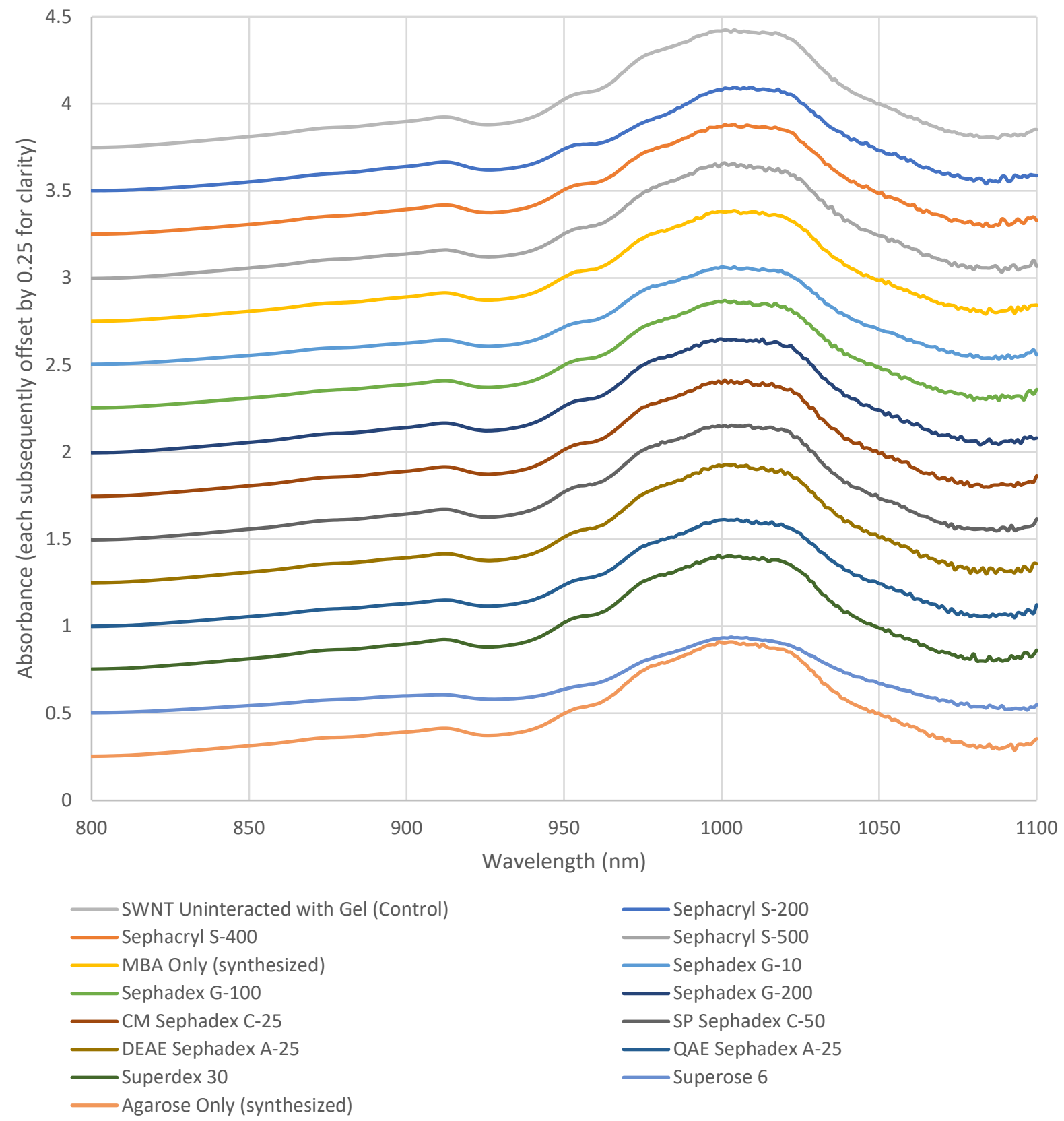

Figure S4. The absorbance spectra of the SWNT before interaction with the gel, and the spectra of the SWNT samples after interaction with each gel, offset for clarity. 


\section{S6. Results from 15-column iterative purification of SWNT using Sephacryl S200, Sephacryl S500, and Superose 6}

While the main body of this work includes a comparison between the multicolumn purification of SWNT enabled by hydrogel materials Sephacryl S200, Sephacryl S500, and Superose 6, those data were truncated after 10 iterative columns because the vast majority of relevant information was contain within that truncated dataset. Here, in the interest of transparency, we present the untruncated 15-column version of that dataset in Figure $\mathbf{S 5}$.
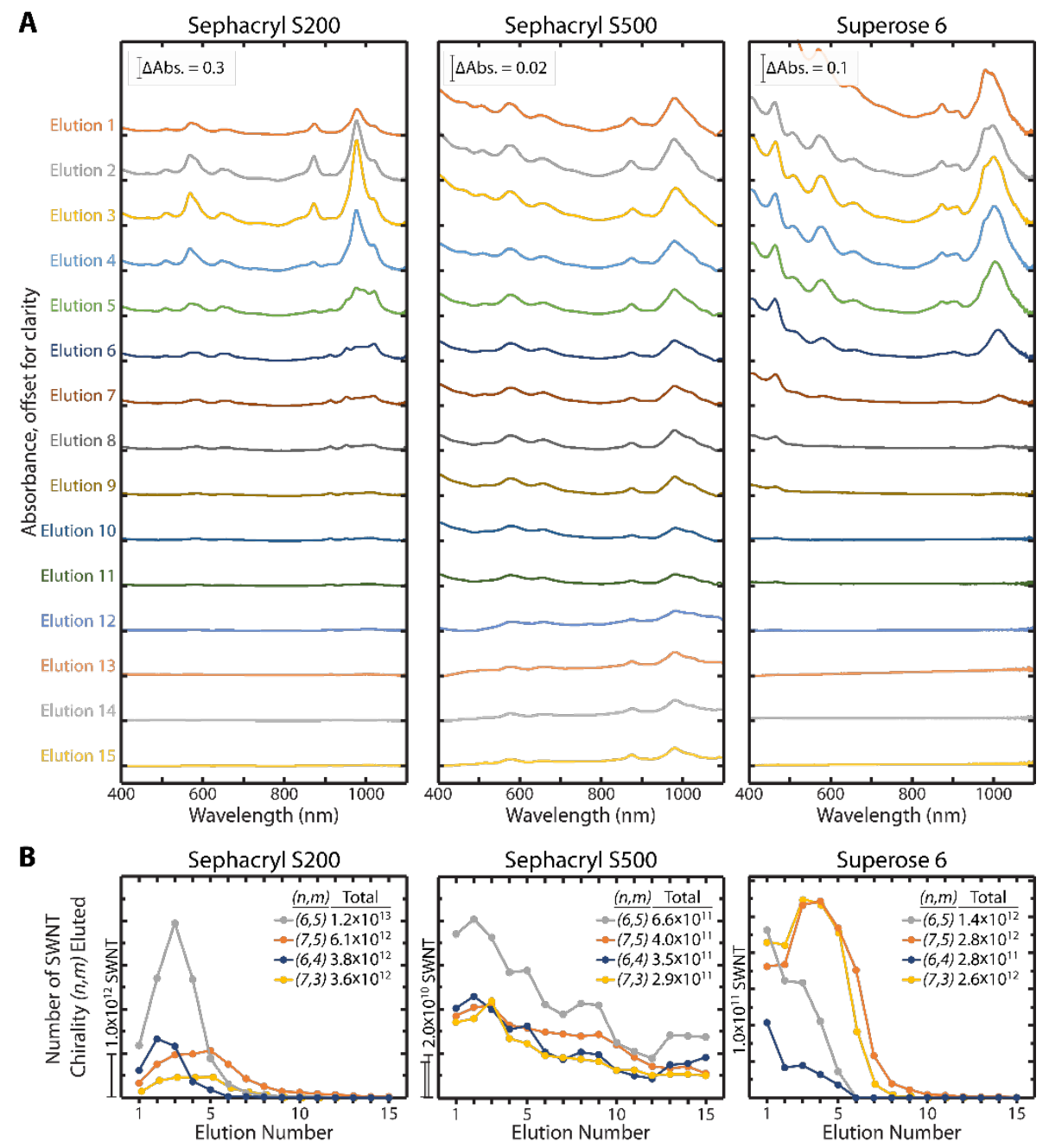

Figure S5. Fifteen column interaction results for Sephacryl S-200, Sephacryl S-500, and Superose 6. (A) shows the absorbance spectrums for the elutions of all fifteen columns, offset for clarity, and (B) shows the total number of SWNT of the specific chiralities $(6,5),(7,5),(6,4)$, and $(7,3)$ eluted from each iterative column. 


\section{S7. Supporting Information References}

(1) Dolan, M.; Watts, B. P.; Tvrdy, K. Tailored Synthesis of Hydrogel Media for Chirality Separation of Single Walled Carbon Nanotubes. Carbon 2021, 171, 597-609. https://doi.org/10.1016/j.carbon.2020.08.074.

(2) Liu, H.; Nishide, D.; Tanaka, T.; Kataura, H. Large-Scale Single-Chirality Separation of Single-Wall Carbon Nanotubes by Simple Gel Chromatography. Nat Commun 2011, 2 (1), article no. 309. https://doi.org/10.1038/ncomms1313.

(3) Watts, B. P.; Barbee, C. H.; Tvrdy, K. Exploiting the Physiochemical Interactions between SingleWalled Carbon Nanotubes and Hydrogel Microspheres To Afford Chirally Pure Nanotubes. ACS Appl. Nano Mater. 2019, 2 (6), 3615-3625. https://doi.org/10.1021/acsanm.9b00567.

(4) Tvrdy, K.; Jain, R. M.; Han, R.; Hilmer, A. J.; McNicholas, T. P.; Strano, M. S. A Kinetic Model for the Deterministic Prediction of Gel-Based Single-Chirality Single-Walled Carbon Nanotube Separation. ACS Nano 2013, 7 (2), 1779-1789. https://doi.org/10.1021/nn305939k.

(5) Jain, R. M.; Tvrdy, K.; Han, R.; Ulissi, Z.; Strano, M. S. Quantitative Theory of Adsorptive Separation for the Electronic Sorting of Single-Walled Carbon Nanotubes. ACS Nano 2014, 8 (4), 3367-3379. https://doi.org/10.1021/nn4058402.

(6) Erickson, H. P. Size and Shape of Protein Molecules at the Nanometer Level Determined by Sedimentation, Gel Filtration, and Electron Microscopy. Biol Proced Online 2009, 11 (1), 32-51. https://doi.org/10.1007/s12575-009-9008-x.

(7) Song, C.; Wang, P.; Makse, H. A. A Phase Diagram for Jammed Matter. Nature 2008, 453 (7195), 629-632. https://doi.org/10.1038/nature06981.

(8) Naumov, A. V.; Ghosh, S.; Tsyboulski, D. A.; Bachilo, S. M.; Weisman, R. B. Analyzing Absorption Backgrounds in Single-Walled Carbon Nanotube Spectra. ACS Nano 2011, 5 (3), 1639-1648. https://doi.org/10.1021/nn1035922.

(9) Kataura, H.; Kumazawa, Y.; Maniwa, Y.; Umezu, I.; Suzuki, S.; Ohtsuka, Y.; Achiba, Y. Optical Properties of Single-Wall Carbon Nanotubes. Synthetic Metals 1999, 103 (1-3), 2555-2558. https://doi.org/10.1016/S0379-6779(98)00278-1.

(10) Sanchez, S. R.; Bachilo, S. M.; Kadria-Vili, Y.; Lin, C.-W.; Weisman, R. B. (N,m)-Specific Absorption Cross Sections of Single-Walled Carbon Nanotubes Measured by Variance Spectroscopy. Nano Lett. 2016, 16 (11), 6903-6909. https://doi.org/10.1021/acs.nanolett.6b02819.

(11) Jain, R. M.; Howden, R.; Tvrdy, K.; Shimizu, S.; Hilmer, A. J.; McNicholas, T. P.; Gleason, K. K.; Strano, M. S. Polymer-Free Near-Infrared Photovoltaics with Single Chirality $(6,5)$ Semiconducting Carbon Nanotube Active Layers. Adv. Mater. 2012, 24 (32), 4436-4439. https://doi.org/10.1002/adma.201202088. 